\title{
Spinal Muscular Atrophy Type 4: A Case Report
}

\author{
G Kalcev* and I Barbov \\ University Clinic for Neurology, Skopje, Republic of North Macedonia \\ *Corresponding Author: G Kalcev, University Clinic for Neurology, Skopje, Republic of North Macedonia.
}

Received: October 14, 2019; Published: November 13, 2019

DOI: 10.31080/ASNE.2019.02.0124

\begin{abstract}
Spinal muscular atrophy is a genetic disorder characterized by weakness and wasting (atrophy) in muscles used for movement (skeletal muscles). It is caused by a loss of specialized nerve cells, called motor neurons that control muscle movements. Mutations in the SMN1 gene cause all types of spinal muscular atrophy. Here we report a 61-year old patient from the Republic of North Macedonia, who was diagnosed with Spinal muscular atrophy type 4. He was admitted to University Clinic in Skopje because of muscle weakness in both legs, without a clear beginning and with slow progression. Muscle weakness was the reason for difficulties in performing basic activities, such as going down the stairs. The finding of EMG was an indicator of a chronic, partial, neurogenic lesion with primary axonal suffering. The magnetic resonance of the spine detected easy-degree, degenerative changes in the lumbosacral part with the initial formation of spondylophytes. Last, but not least, molecular genetic analysis showed the absence of the SMN1 gene in a homozygous state (0 copies SMN1) and the presence of increased copy number of SMN2 pseudogene (> 5 copies SMN2). This finding confirmed the acute form of adult spinal muscular atrophy (type 4). By presenting this case, we have tried to get you more closely to the importance of this rare neuromuscular disease.
\end{abstract}

Keywords: Neuromuscular Disorders; Muscle Weakness; Spinal Muscular Atrophy Type IV

\section{Introduction}

Spinal muscular atrophy type IV (SMA4) is an adult-onset, a mild form of SMA. It is a rare disease with a prevalence of $1 / 300,000$. SMA4 is usually manifested in the second or third decade of life. According to the definition, it is a motor neuron disease, accompanied by proximal muscle weakness with lower motor neuron features $[1,2]$. SMA4 is s a rare genetic neuromuscular disorder that results in progressive muscle weakness and atrophy. Generally, it is a fatal disease due to the involvement of the respiratory muscles leading to respiratory failure [3]. However, the presence of sensory and sphincter abnormalities and signs of the upper motor neuron should raise suspicion of other associated conditions [4].

\section{Case Report}

A 61-year old patient was admitted to University Clinic in Skopje, Republic of North Macedonia because of muscle weakness in both legs, without a clear beginning and with slow progression. Muscle weakness was the reason for difficulties in performing basic activities, such as going down the stairs. The rough motor power of the upper and lower limbs was reduced, indicated on proximal muscles. In addition to this, it was noticed reduced reflex responses of arms and lost reflex responses of the legs. Otherwise, there were visible fasciculations of the left deltoid muscle, after percussion provocation. Gowers' sign was positive. Furthermore, a high value of creatinine kinase was observed, with a value of $289 \mathrm{U} / \mathrm{L}$ (range $22 \mathrm{U} / \mathrm{L}$ to $198 \mathrm{U} / \mathrm{L}$ ). The patient gave data that all male members of his family were/are with weak legs. Other diseases observed in the patient were Diabetes mellitus type 2 and hyperlipidemia. Concerning the findings of electromyography, it registered polyphase, prolonged, low voltage motor unit action potentials in both thighs at voluntary contractions, as well as high-voltage motor unit action 
potentials in all remaining muscles in the arms and legs. Clearly, this finding of EMG was an indicator of a chronic, partial, neurogenic lesion with primary axonal suffering. The magnetic resonance of the spine detected easy-degree, degenerative changes in the lumbosacral part with the initial formation of spondylophytes. At L5-S1 level, dorsal protrusion of the intervertebral disc with compression of the dural saccus, the neural openings and the cauda were detected. Last, but not least, molecular genetic analysis showed the absence of the SMN1 gene in a homozygous state 0 copies SMN1) and the presence of increased copy number of SMN2 pseudogene ( $>5$ copies SMN2). This finding confirmed the acute form of adult spinal muscular atrophy (type 4). Genetic testing was recommended.

\section{Discussion}

Spinal muscular atrophy is a rare neuromuscular disease that is characterized by degeneration of the frontal horn cells in the spinal cord and motor nuclei in the lower brainstem. It results in progressive muscle weakness and atrophy [3]. Right now, SMA disorders are classified based on the age of onset and clinical course into four types (zero to four) [5]. Muscle weakness primarily affects the legs and hip muscles and then progresses to the shoulders and arms. The clinical picture is similar to that of SMA3, but motor weakness occurs in a milder form in SMA4 [6,9]. SMA type four is the softest form of SMA disorders. It occurs in adults with a diffuse, symmetric weakness that affects the proximal muscles, with absent or significantly reduced deep tendon reflexes. Electromyography and muscle biopsy were standard tools in the diagnostic procedure for this disease. Nowadays, molecular genetic testing is considered as the gold standard procedure in confirmation in the diagnosis of SMA4, by detection of homozygous deletions or mutations of the SMN1 gene [5]. SMA4 is inherited autosomal recessively, and its gene, the SMN1 gene is located on chromosome 5q13.2. Deficiency with the SMN1 gene occurs when bi-allelic deletions or mutations are found in this gene. As a result, patients who have a single intact copy of the SMN1 gene are in fact carriers of the disease, without manifesting symptoms $[5,10]$. Although there are some differences, the severity of the disease depends on the number of copies of the second SMN gene (SMN2). Almost all patients have a normal life expectancy [7]. Until 2016, the treatment for this disease was supportive. A new therapeutic option, Nusinersen (Spinraza) became available for patients with SMA in December 2016. Nusinersen is an oligonucleotide that has the role to increase the expression of the survival motor neuron protein. It is administered in the cen- tral nervous system (CNS) using intrathecal injection for four initial loading doses; the first three loading doses are given at 14-day intervals, while the fourth loading dose is given 30 days after the third. After that, a maintenance dose is given every four months [8].

\section{Conclusion}

By presenting this case, we have tried to emphasize and get you more closely to the importance of this rare disease. Molecular genetic testing, as well as treatment, are the key factors for halting the progression of the symptoms of this disease.

\section{Conflict of Interest}

There is not a conflict of interest. Permission to publish this case report was obtained from the patient.

\section{Founding}

None.

\section{Bibliography}

1. Prior TW and Russman BS. "Spinal muscular atrophy". Gene Reviews (2014).

2. Lunn MR and Wang CH. "Spinal muscular atrophy". Lancet 371.9630 (2008): 2120-2133.

3. Kolb SJ and Kissel JT. "Spinal muscular atrophy". Neurologic Clinics 33 (2015): 831-846.

4. Peeters K., et al. "Clinical and genetic diversity of SMN1-negative proximal spinal muscular atrophies". Brain 137.11 (2014): 2879-2896.

5. Arnold WD., et al. "Spinal muscular atrophy: Diagnosis and management in a new therapeutic era". Muscle Nerve 51 (2015): 157-167.

6. Kroksmark AK., et al. "Muscle strength and motor function in children and adolescents with spinal muscular atrophy II and III". European Journal of Paediatric Neurology 5 (2001): 191-198.

7. Hauke J., et al. "Survival motor neuron gene 2 silencing by DNA methylation correlates with spinal muscular atrophy disease severity and can be bypassed by histone deacetylase inhibition". Human Molecular Genetics 18 (2009): 304-317.

8. Drugs @ FDA: FDA Approved Drug Products (2016). 
9. Haaker G and Fujak A. "Proximal spinal muscular atrophy: Current orthopedic perspective". The Application of Clinical Genetics 6 (2013): 113-120.

10. Sedghi M., et al. "Genotype-phenotype correlation of survival motor neuron and neuronal apoptosis inhibitory protein genes in spinal muscular atrophy patients from Iran". Advanced Biomedical Research 3 (2014): 74.

Volume 2 Issue 12 December 2019

(C) All rights are reserved by G Kalcev and I Barbov. 